\title{
Core-hole delocalization for modeling X-ray spectroscopies: A cautionary tale
}

\author{
Iulia Emilia Brumboiu ${ }^{1}$ and Thomas Fransson ${ }^{2}$ \\ 1) Department of Chemistry, Pohang University of Science and Technology (POSTECH), 37673 Pohang, \\ Republic of Korea \\ ${ }^{2)}$ Department of Theoretical Chemistry and Biology, KTH Royal Institute of Technology, 10691 Stockholm, \\ Sweden
}

The influence of core-hole delocalization for X-ray photoelectron, X-ray absorption, and X-ray emission spectrum calculations is investigated in detail, using approaches including response theory, transition-potential methods, and ground state schemes. The question of a localized/delocalized vacancy is relevant for systems with symmetrically equivalent atoms, as well as near-degeneracies which can distribute the core-orbitals over several atoms. We show that issues relating to core-hole delocalization are present for calculations considering explicit core-hole states, e.g. when using a core-excited or core-ionized reference state, or for fractional occupation numbers. Including electron correlation eventually alleviates the issues, but even using $\operatorname{CCSD}(\mathrm{T})$ there is a noticable discrepancy between coreionization energies obtained with a localized and delocalized core-hole $(0.5 \mathrm{eV}$ for the carbon $K$-edge). Within density functional theory, the discrepancy is associated with the exchange interaction involving the core orbitals of the same spin symmetry as the delocalized core-hole, and the size of the error is thus directly related to the amount of HF exchange included in the functional. For linear response methods, we further show that if X-ray absorption spectra are modelled by considering symmetry-unique sets of atoms, care has to be taken such that there are no delocalizations of the core orbitals, which would otherwise introduce shifts in absolute energies and relative features.

\section{INTRODUCTION}

When modeling X-ray spectroscopies, the question of corehole $(\mathrm{CH})$ localization can be highly influential on the integrity of the final spectra. Using a $\triangle \mathrm{SCF}$ approach, it has long been noted that a localized $\mathrm{CH}$ results in ionization energies (IE:s) in good agreement with experiments, while a delocalized CH overestimates these IE:s. ${ }^{1}$ Subsequently, when decomposing the terms involved in relaxing a core-hole state, ${ }^{2}$ it was shown that the relaxation effects associated with a localized core-hole are analogous to relaxation and correlation effects associated with a delocalized core-hole. ${ }^{3}$ As such, uncorrelated $\triangle \mathrm{SCF}$ works quite well when the $\mathrm{CH}$ is localized, but not when it is delocalized. Note that the use of the term correlation in this context can be somewhat misleading, as the deficiency of the delocalized $\mathrm{CH}$ has more to do with inadequate orbitals which are then corrected through correlation. ${ }^{4}$ Nevertheless, these terms are still relatively descriptive and commonly used in the literature, but some care is warranted. Using a localized $\mathrm{CH}$, the symmetry of the molecular system is broken, but it can be retrieved by forming linear combinations of the localized solutions. ${ }^{1}$ Alternatively, by including electron correlation via various post-HF methods, theoretical results in good agreement with experimental spectra can be achieved while preserving the full symmetry of the system. ${ }^{5,6}$

From a more fundamental point of view, the localization of core-holes has been investigated and discussed experimentally, with measurements observing both localized and delocalized $\mathrm{CHs}$, depending on the manner of conducting the measurements. It has thus been seen that $\mathrm{CH}$ localization/delocalization can be an effect of the observation (a situation which is not too uncommon in quantum physics). ${ }^{7-11}$ In certain cases, an entangled system is probed, while under other conditions the entanglement is broken and an independent particle picture emerges. Care must thus be taken when performing the analysis of these types of measurements, where an overly simplistic single-particle picture should be avoided in lieu of more complete quantum mechanical frameworks.

While the fundamental question of $\mathrm{CH}$ localization can be imperative for gaining a deeper insight into the underlying physics, for most practical spectrum calculations it may not be as vital. Still, it remains important to determine how $\mathrm{CH}$ localization/delocalization affects the agreement of calculated spectra to experiment or other computations, and this is the goal of the present study. As will be discussed further, the use of a delocalized $\mathrm{CH}$ introduces an error - which we here designates as the delocalization error - found to be associated to the number of delocalization sites, ${ }^{12}$ as relates to early studies on the core-hole screening effects for atoms. ${ }^{13}$ The relaxation effects were there shown to be quadratic with the change in shielding constant, and thus inversely proportional to the number of sites. Studies on delocalization errors have been conducted for ionization energies, ${ }^{12,14}$ potential energy surfaces, ${ }^{4}$ X-ray Raman scattering, ${ }^{15}$ and resonant inelastic $\mathrm{X}$-ray scattering, ${ }^{16}$ to name a few. The behaviour of the delocalization error in HF compared to DFT has been noted, with the error typically having opposite sign. ${ }^{17}$ A number of measures to remove the error have been proposed and include the above mentioned linear combination of localized solutions, ${ }^{1}$ the use of high-level wave function theory, ${ }^{5,6}$ as well as tailoring various specialized methodologies. For example, a $Z+Q$ model has been formulated to replace the $Z+1$ (or equivalent core) approximation for cases where the $\mathrm{CH}$ is delocalized. ${ }^{18}$ Furthermore, $\mathrm{CH}$ localization has been analyzed for the static-exchange (STEX) approximation description of inner-shell photoionization. ${ }^{19}$

The issue of $\mathrm{CH}$ localization has also been discussed in the context of double core-hole (DCH) spectroscopy and other multi-electron processes. Here, the creation of core- 
holes at different atomic sites typically yields superior sensitivity to the local chemical environment when compared to single core-hole approaches. In this context, $\mathrm{CH}$ localization/delocalization may have an even stronger influence than in single core-hole spectra, as discussed in a seminal article by Cederbaum et al. ${ }^{20}$ If a delocalized scheme is used, the correlation effects become substantial and need to be accounted for. A localized approach is thus the more common way of considering DCH processes. ${ }^{21-23}$ Considering multi-ionized $\mathrm{N}_{2}$, it has been noted that the difference between a localized and a delocalized picture depends on the number of holes created with an even number of holes the difference is close to zero, but for uneven number of holes it becomes substantial. ${ }^{24}$

In this work, we investigate the influence of $\mathrm{CH}$ delocalization for a number of X-ray spectroscopies, as modeled using several different methods. In recent years, illustrations on the practical impact of using a delocalized $\mathrm{CH}$ have been shown for, e.g., single and double core hole X-ray emission spectroscopy, ${ }^{23,25}$ and core-excitation calculations with restricted open-shell DFT. ${ }^{26}$ We here seek to provide a more systematic overview of where $\mathrm{CH}$ delocalization errors occur, as well an analysis of the involved terms. Importantly, focus will not only be on absolute energies, but also on relative energies and intensities. Related, the impact of near-degeneracies for nonequivalent sites when considering XAS is also addressed, with focus on approaches in which each symmetrically equivalent set of atomic sites is considered individually in order to decrease computational costs. This type of approach has been successfully applied for, e.g., CVS-ADC, ${ }^{27,28}$ and we here go into more detail into its potential pitfalls.

\section{THEORY AND METHODS}

This section includes a brief overview of the methods considered in this article, whereas a more extensive discussions on modeling X-ray spectroscopies can be found in, e.g., Ref. 29.

\section{A. Converging a core-hole state}

When constructing a $\mathrm{CH}$ reference state, some constraint has to be imposed in order to ensure that the SCF optimization does not collapse into a valence-ionized state. One of the approaches which can be used to achieve this is the maximum overlap method (MOM) ${ }^{30,31}$ In MOM, the wave function is optimized with overlap to previous iterations in mind, rather than from energetic arguments. With this non-Aufbau procedure, core-holes and other energetically unstable wave functions can be constructed. Alternative approaches of forming a $\mathrm{CH}$ reference state are available, including the initial maximum overlap method (IMOM) ${ }^{32}$ state-targeted energy projection (STEP), ${ }^{33}$ and square gradient minimization (SGM). ${ }^{34}$ Core-hole states constructed this way can be used to estimate ionization energies (IE:s) via the $\triangle \mathrm{SCF}$ approach, i.e. from the difference in energy between the ground state and a coreionized state. $\triangle \mathrm{MP} / \triangle \mathrm{CC}$ IE:s can be obtained in a similar way by using these neutral and core-ionized states as the reference for an MP/CC calculation.

\section{B. Transition potential DFT}

Besides the calculation of core-ionization energies and as an initial state of X-ray emission calculations, core-holes can also be used to include the relaxation effects involved in X-ray absorption spectroscopy (XAS). One example is Slater's transition state method which models relaxation by considering a half-empty core orbital in combination with the addition of half an electron to each probed unoccupied MO..$^{35,36}$ The total spectrum is then constructed by calculating orbital energy differences and transition matrix elements, but the approach requires one explicit calculation for each final state. A further simplification of the method is to relax the electronic structure in the presence of a partial core-hole, constructing the full spectrum from a single Hamiltonian. Within a DFT framework, this approach constitutes the transition potential DFT (TP-DFT) method, ${ }^{37,38}$ which commonly employs a half core hole $(\mathrm{HCH})$, although other occupations are also used..$^{39,40} \mathrm{By}$ introducing a shift such that the eigenvalue of the core level is equal to the calculated IE, TP-DFT provides XAS spectra that compare well to experiment in many cases, ${ }^{37,38,41}$ albeit with some occasional difficulties in sufficiently capturing relaxation effects, ${ }^{39}$ and relatively large spread in relative energies. ${ }^{28}$ More recent developments related to this method include the combination of the TP-DFT philosophy with coupled cluster, yielding the transition potential coupled cluster (TP-CC) method. ${ }^{42}$

Alternatively, an approach using the energy difference and transition dipole moments between the ground state molecular orbitals has been used, with good relative agreement to experiment. ${ }^{43,44}$

\section{Linear response methods}

In spite of successful application to large molecules ${ }^{45-47}$ and extended systems, ${ }^{48-50}$ TP-DFT is essentially a ground state theory for XAS. A different approach is to use the equation of motion describing the linear response of the electron system to an applied electromagnetic field. ${ }^{51}$ In timedependent HF (TDHF) and time-dependent DFT (TDDFT) this is achieved through the random phase approximation (RPA) equation. ${ }^{52,53}$ Computational costs can be lowered by using the Tamm-Dancoff approximation (TDA), ${ }^{54}$ which for TDHF becomes equivalent to configuration interaction singles (CIS).$^{53}$ In TDDFT, the approximate nature of the exchange and correlation $(\mathrm{xc})$ functional leads to self-interaction errors (SIE) ${ }^{29,55-57}$ that are exacerbated in the case of coreexcitations (due to the high densities in the core region). This has spurred the design of a plethora of tailored xcfunctionals, ${ }^{29,56,58}$ which can yield improved absolute energies, but not necessarily improved relative energies. ${ }^{28}$

Among correlated $a b$ initio methods for excited states, of interest here are the algebraic diagrammatic construction 
(ADC) scheme for the polarization propagator, and coupled cluster (CC) theory. In ADC, the polarization propagator is expanded in a perturbation series, where the poles and residues of its spectral representation correspond to excitation energies and transition amplitudes, respectively. ${ }^{59-63}$ The hierarchy of ADC methods is obtained by truncating the perturbation expansion at desired order, with efficient implementations available for $\mathrm{ADC}(1), \mathrm{ADC}(2), \mathrm{ADC}(2)-\mathrm{x}$, and $\mathrm{ADC}(3 / 2)$. Note that $\mathrm{ADC}(1)$ is equivalent to CIS in terms of transition energies. An alternative hierarchy of post-HF methods is available through the coupled cluster (CC) approach, which can be considered either in an equation-ofmotion $(\mathrm{EOM})^{64,65}$ or linear response (LR) ${ }^{66-68}$ formalism. For XAS, ADC(2)-x and (EOM-)CCSD have been shown to yield good agreement to experiment, ${ }^{28,69,70}$ while for X-ray emission spectra good absolute agreement with experiments is provided by ADC(2) and EOM-CCSD (with ADC(2)-x yielding good relative features). ${ }^{56,71-73}$

When calculating X-ray absorption spectra, a flavour of the core-valence separation (CVS) approximation must typically be applied. ${ }^{29,69,70,74-78} \mathrm{CVS}$ avoids the convergence of a great manifold of valence-excited and valence-ionized states by utilizing the fact that the coupling between core- and valenceexcited states is small, motivating the decoupling of coreexcitations from the total manifold. The precise implementation of this scheme varies between electronic structure methods and software packages. For the $K$-edge, the error associated to the CVS approximation in TDHF and TDDFT has been shown to be negligible, while in post-HF methods it has been shown to be small and stable over different compounds. ${ }^{76,77,79,80}$

When it comes to X-ray emission spectra, they involve the use of a core-hole reference state for which the valence-tocore transitions occur as the first (negative) eigenvalues. ${ }^{56,71,72}$ XES calculations have successfully been performed using TDDFT, ${ }^{56,81,82}$ EOM-CCSD, ${ }^{56,71,81}$ and ADC, ${ }^{72,73}$ where we note that the relaxation (and thus the performance of different methods) is markedly different compared to that of coreexcitation processes. ${ }^{72}$

\section{COMPUTATIONAL DETAILS}

The molecular geometries were optimized at the frozencore MP2 $2^{83} / \mathrm{cc}-\mathrm{pVTZ}{ }^{84}$ level of theory, using the Q-Chem 5.2 program. ${ }^{85}$ Property calculations were performed using cc-pVDZ for hydrogen atoms, and aug-cc-pCVTZ for the remaining elements. Core-holes were localized by applying effective core potentials (ECP:s) of the Stuttgart-Cologne type $^{86}$ for all save one atomic site of an investigated element, or in some cases (Figs. 5 and 7) by using a distorted structure. For the delocalized core-hole calculations, the maximum overlap method $(\mathrm{MOM})^{30,31}$ was used to obtain a core-hole with the same symmetry as that of the corresponding ground state core orbital. The smallest absolute values of the MP2 energy denominator for the ethene core-holes were seen to be $\sim 1$ Hartree, and numerical instabilities due to near-singularities are thus not present for these calculations. ${ }^{73}$ The $\mathrm{PBE}^{87,88}$ exchange-correlation functional was used for the DFT calculations, unless otherwise stated. Convolution of the calculated energies and intensities was performed using a Lorentzian function of $0.25 \mathrm{eV}$ half-width at half maximum (HWHM) to facilitate the analysis and comparison of the spectra.

The TDDFT and ADC calculations were carried out in QChem 5.2, ${ }^{85}$ with the restricted energy-window and CVS ${ }^{75,89}$ approaches being applied when computing X-ray absorption spectra. Calculations using tailored CVS spaces were run using the adcc software package, ${ }^{27}$ with SCF results obtained from pyscf. ${ }^{90,91}$ The TDA was applied for TDDFT calculations of emission spectra.

An in-house modified version of the psiXAS plugin module $^{92}$ of $\mathrm{psi}^{93}$ was used for TP-DFT and ground state DFT calculations. For XAS calculations in the TP-DFT framework, the half core-hole $(\mathrm{HCH})$ approximation was used, without any additional shifts in energy. In order to perform XES calculations, we have modified the psiXAS module to also allow the computation of transition dipole moment integrals between the core-hole state and occupied orbitals (rather than unoccupied orbitals as required by core-excitation calculations). This modification was also used to determine the transition dipole moments between ground state orbitals, which together with MO energy differences yields spectra within ground state (GS) DFT.

\section{RESULTS AND DISCUSSION}

We first illustrate the effects of core-hole delocalization in XAS, XES, and IE/XPS calculations. The influence of various xc-functionals, probed element, and system size is then discussed, followed by a breakdown of energy contributions in DFT. The impact of electron correlation in a post-HF framework is then considered, together with some general recommendations for calculations involving explicit core-holes. Finally, we consider the use of a tailored CVS space, including cases of fully symmetric atomic sites, near-degeneracy, and localized core-orbitals.

\section{A. Influence of core-hole delocalization}

The effects of using localized or delocalized CH:s are illustrated Fig. 1, considering X-ray absorption and emission spectra using five different methods. In the case of XAS, a delocalized $\mathrm{CH}$ yields the same spectra for all methods save TPDFT, for which a static shift of $8.39 \mathrm{eV}$ is introduced. Only minor differences in relative spectrum features are noted following this energy shift. The difference in transition energies is due to the use of an explicit (fractional) core-hole in TPDFT, something which is not present for the other approaches. Comparing to experimental measurements, the $1 s \rightarrow \pi^{*}$ peak is situated at $284.7 \mathrm{eV},{ }^{94}$ which is close to the localized results.

For XES, significant differences in both absolute energies and relative features are noted for all methods except using DFT ground state MOs, which is the only approach not using an explicit $\mathrm{CH}$ reference state. The largest differences in 

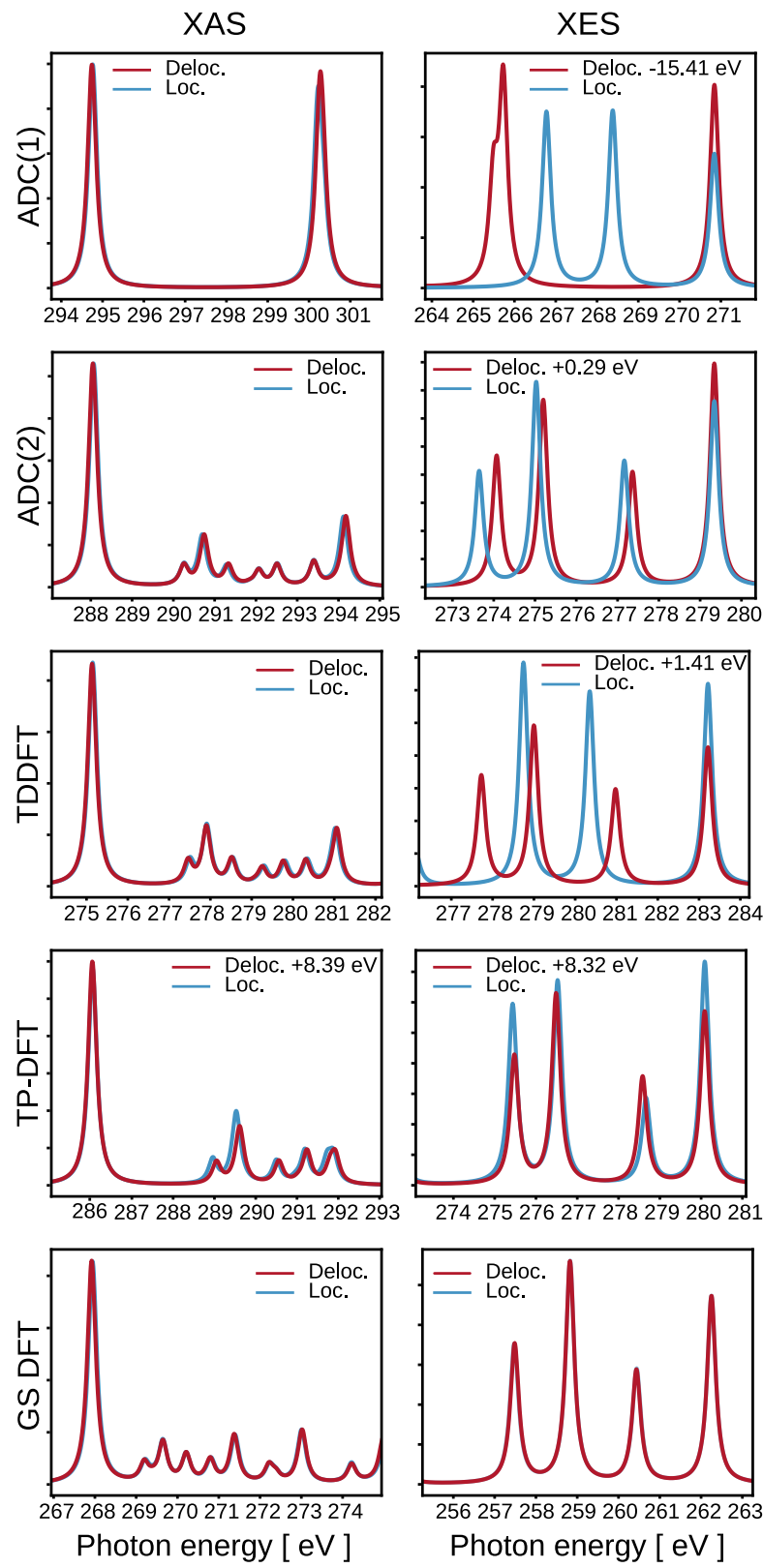

FIG. 1. X-ray absorption (left) and X-ray emission (right) spectra of ethene calculated at different levels of theory, using a localized (blue) or delocalized (red) core-hole. Where necessary, delocalized $\mathrm{CH}$ spectra have been shifted to align with the corresponding localized $\mathrm{CH}$ spectrum, using rigid shifts in energy.

both absolute and relative features are observed for TDDFT and ADC(1), as these methods do not include the highly influential relaxation effects involved in core properties - the difference observed between a localized and a delocalized $\mathrm{CH}$ is ultimately a consequence of the improper treatment of electron correlation and orbital relaxation. ${ }^{2}$

The distinction between electron correlation and relaxation is somewhat arbitrary, ${ }^{3,95}$ with relaxation for core properties often being included through electron correlation. ${ }^{28,29,96}$ Still, the distinction can be helpful for analysis, and in Fig. 2 we use these designations in order to better illustrate the various contributions for calculations using HF and DFT. Included here are results for IE:s, and one feature each of XES and XAS, as compared to reference calculations using $\operatorname{CCSD}(\mathrm{T})$. The reference calculations are all performed considering different HF reference states (neutral ground state, core-ionized, neutral core- and valence-excited), while the XES/XAS results for HF and DFT have been obtained within the Tamm-Dancoff approximation. For the CCSD(T) reference calculations, it is clear that the use of a localized or delocalized $\mathrm{CH}$ yields very similar results, with ionization or transition energies varying by $0.40-0.54 \mathrm{eV}$ (larger for a delocalized $\mathrm{CH}$ ). This is primarily a result of CCSD(T) still lacking some electron correlation, with minor contributions also stemming from the use of ECP:s when localizing the CH. Note that the CCSD(T) calculations of the final state in XAS is not spin-pure, but the resulting energies are similar to those obtained by, e.g., CVSADC(2)-x or CVS-EOM-CCSD, and are thus expected to be reasonably good.

Moving to the HF results, approximately $11 \mathrm{eV}$ of correlation energy $\left(\mathrm{E}_{\mathrm{c}}\right)$ is lacking for the initial states of IE, XAS, and for the localized description of XES. For the final states, there is generally a lack of both relaxation and correlation $\left(E_{\mathrm{rc}}\right)$, although for the $\Delta \mathrm{HF}$ calculations of IE:s the relaxation can, potentially, be accounted for separately (yielding $\mathrm{E}_{\mathrm{r}}$ ). This is seen for the localized $\mathrm{CH}$, where a lowering in final state energy of $15.26 \mathrm{eV}$ is achieved when compared to that obtained using Koopmans' theorem (dashed lines). Only approximately half of this relaxation energy $(7.38 \mathrm{eV})$ is retrieved if a delocalized $\mathrm{CH}$ is used, yielding a remaining 19.77 $\mathrm{eV} \mathrm{E}_{\mathrm{rc}}$, and an overestimation of the final ionization energy of about $7 \mathrm{eV}$. This partial relaxation $\left(\mathrm{E}_{\mathrm{r}},\right)$ is in line with previous observation, where the factor one half comes from the relation of relaxation for a localized $\mathrm{CH}$ being analogous to correlation and relaxation of a delocalized $\mathrm{CH}$, and the introduced discrepancy being inversely proportional to the number of delocalization sites. ${ }^{3,12,13}$ For XES, the final state lacks both relaxation and correlation (when using TDHF-for $\triangle \mathrm{HF}$ the situation would be closer to that of IE:s), and is thus further from the CCSD(T) reference than the $\sim 11 \mathrm{eV} \mathrm{E} \mathrm{E}_{\mathrm{c}}$ of most initial states. For a localized $\mathrm{CH}$, the energy difference between the initial and final state is thus too low, and the emission energy is approximately $10 \mathrm{eV}$ lower compared to $\operatorname{CCSD}(\mathrm{T})$. For a delocalized $\mathrm{CH}$ the situation is quite different: the initial state has only partial relaxation accounted for $\left(\mathrm{E}_{\mathrm{r}^{\prime}}\right)$, and is thus $19.77 \mathrm{eV}$ above the reference energy. The final state again lacks $\mathrm{E}_{\mathrm{rc}}$, but this is in total smaller than for a localized $\mathrm{CH}$ due to the use of a poorer (and thus more GS-like) initial state. The resulting emission energy therefore overestimates the reference values by almost $6 \mathrm{eV}$, yielding a large discrepancy when compared to the localized description. For XAS, no explicit core-hole is used, and the localized and delocalized results are thus very similar, both featuring an overestimation in transition energy of approximately $9 \mathrm{eV}$, due to the unrelaxed (and thus high-energy) final state.

Moving to the DFT results with a hybrid xc-functional (25\% HF exchange), the situation is a bit different on account of the (partial) inclusion of correlation, as well as the presence 


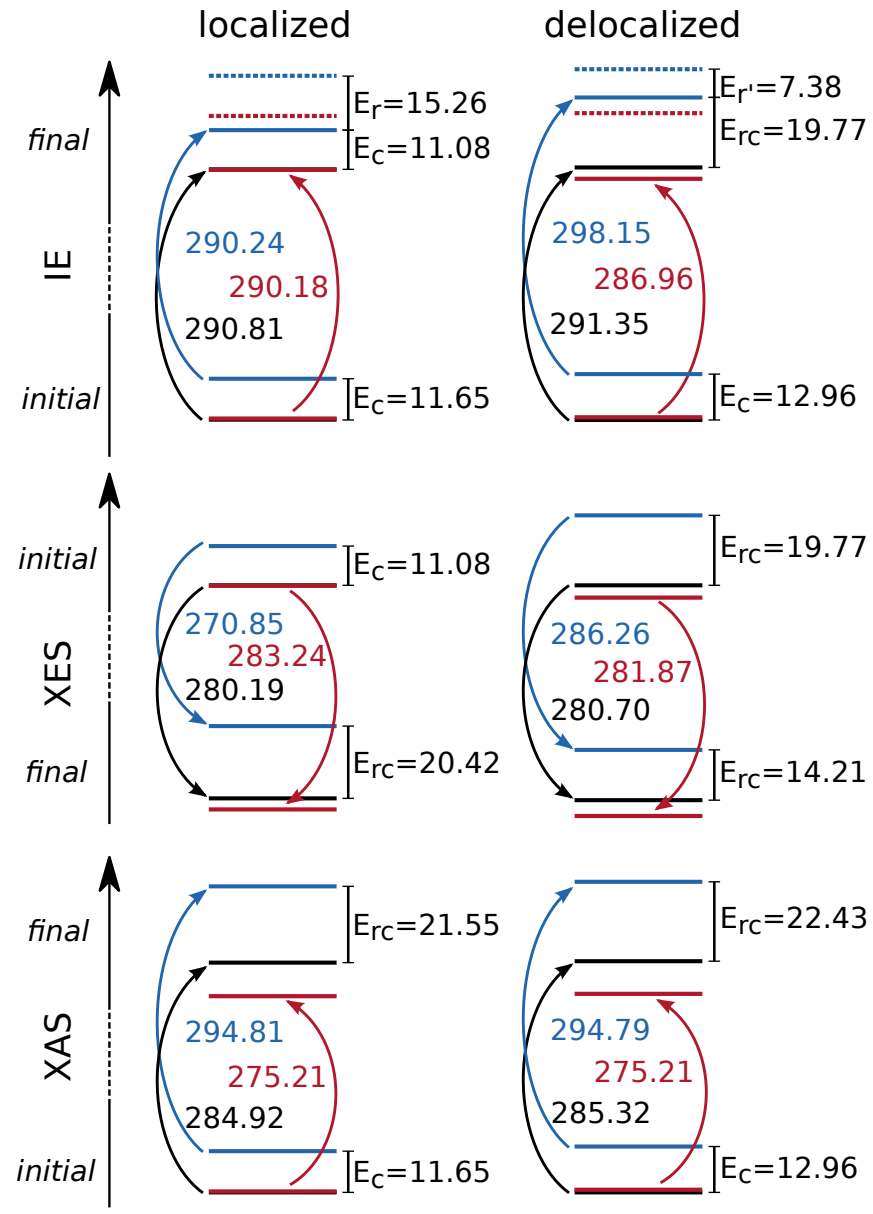

FIG. 2. Initial and final state energies for carbon 1s ionization energy (IE), highest feature of the X-ray emission spectrum (XES), and lowest feature of the X-ray absorption spectrum (XAS), as obtained for ethene using a localized or delocalized core-hole. Reference CCSD(T) results shown in black, and HF and PBE0 results in blue and red, respectively, including resulting ionization and transition energies For IE:s, the binding energy from Koopmans' theorem is also shown (dashed lines). Right side of horizontal lines showing discrepancy of HF results compared to CCSD(T) values (or HF values for binding energies from Koopmans' theorem), which is associated with lacking correlation $\left(\mathrm{E}_{c}\right)$, relaxation $\left(\mathrm{E}_{r}\right)$, partial relaxation $\left(\mathrm{E}_{r^{\prime}}\right)$, and combined relaxation and correlation $\left(\mathrm{E}_{r c}\right)$. All energies are expressed in $\mathrm{eV}$.

of self-interaction errors (SIE:s). ${ }^{29,55-57}$ The SIE:s will generally decontract high-density orbitals, and will partially cancel out the effects of lacking relaxation. This is most clearly seen for XAS, where we obtain identical results with a localized or delocalized $\mathrm{CH}$, both underestimating the $\operatorname{CCSD}(\mathrm{T})$ reference by almost $10 \mathrm{eV}$ (despite the initial state energy being very close to that of CCSD(T)). For IE:s, the localized CH leads to results well in line with the reference, while the delocalized $\mathrm{CH}$ lowers the total IE by about $2 \mathrm{eV}$. This difference when compared to HF will discussed more below. Finally, for XES, DFT yields a too large energy difference between the initial and final states in both the localized and delocalized cases, thus resulting in emission energies which are too high. As the initial state is a bit too low for a delocalized $\mathrm{CH}$, the final emission energy is here actually closer to reference.

\section{B. Dependence on element, exchange-correlation functional, and system size}

Considering the dependence of the delocalization error (here defined as the difference to a localized $\mathrm{CH}$ ), with probed atom type and $\mathrm{xc}$-functional, Fig. 3 shows the size of this error for different elements and functionals. The left panel shows the IE discrepancy as a function of $Z$, considering ethene, $\mathrm{N}_{2}, \mathrm{O}_{2}$, and $\mathrm{F}_{2}$. $\mathrm{HF}$ and the PBE functional with varying levels of HF exchange both show an increasing IE discrepancy as $Z$ increases. This simply reflects the increasing relaxation effect and SIE of heavier elements. Focusing on the IE of ethene, the right panel shows the delocalization error of a number of different $\mathrm{xc}$-functionals, grouped into four main categories. For global and range-separated GGA:s the relation between the delocalization error and amount of HF exchange is clear, showing a linear behaviour with increasing HF exchange. This points to the HF exchange parameter being the most influential factor to consider when analysing the performance of DFT for $\mathrm{CH}$ delocalization, as will be discussed further below. By comparison, for global and range-separated meta-GGA:s there are some clear outliers, although the general trend is in line with the GGA:s. The outliers are primarily meta-GGA:s with exchange coefficients that sum up to more than one, with M06-HF, MN15, and MN12-SX deviating the most from the otherwise linear behaviour.

Another parameter which plays a role in the absolute value of the delocalization error is the size of the system over which the $\mathrm{CH}$ is delocalized. This is illustrated in Fig. 4, where the IE error for ethene and cycloalkanes of increasing size is shown as a function of the inverse number of atomic sites over which the $\mathrm{CH}$ is delocalized. The IE:s were calculated for the core MOs which are delocalized over the entire cycloalkanes. Irrespective of the amount of HF exchange included in the functional, the delocalization error increases in absolute value with increasing the number of sites $(N)$. Represented as a function of $1 / N$, the behaviour is almost linear, as expected. ${ }^{12,13}$ Note that what is important is not the actual system size, but rather the number of involved atomic sites.

\section{Energy breakdown within HF and DFT}

To further analyze the behaviour of the delocalization error in HF and DFT, we decompose the different terms involved in calculating the IE, as a function of amount of HF exchange. In $\triangle \mathrm{SCF}$, the IE is calculated as the total energy difference between a core-ionized $\left(E_{\mathrm{CH}}\right)$ state and the ground state $\left(E_{\mathrm{GS}}\right)$ :

$$
\mathrm{IE}=E_{\mathrm{CH}}-E_{\mathrm{GS}} .
$$

Or, decomposed into the one-electron contribution stemming from the Core-Hamiltonian $\left(E_{\mathrm{H}}\right)$, Coulomb $\left(E_{\mathrm{J}}\right)$, HF exchange 

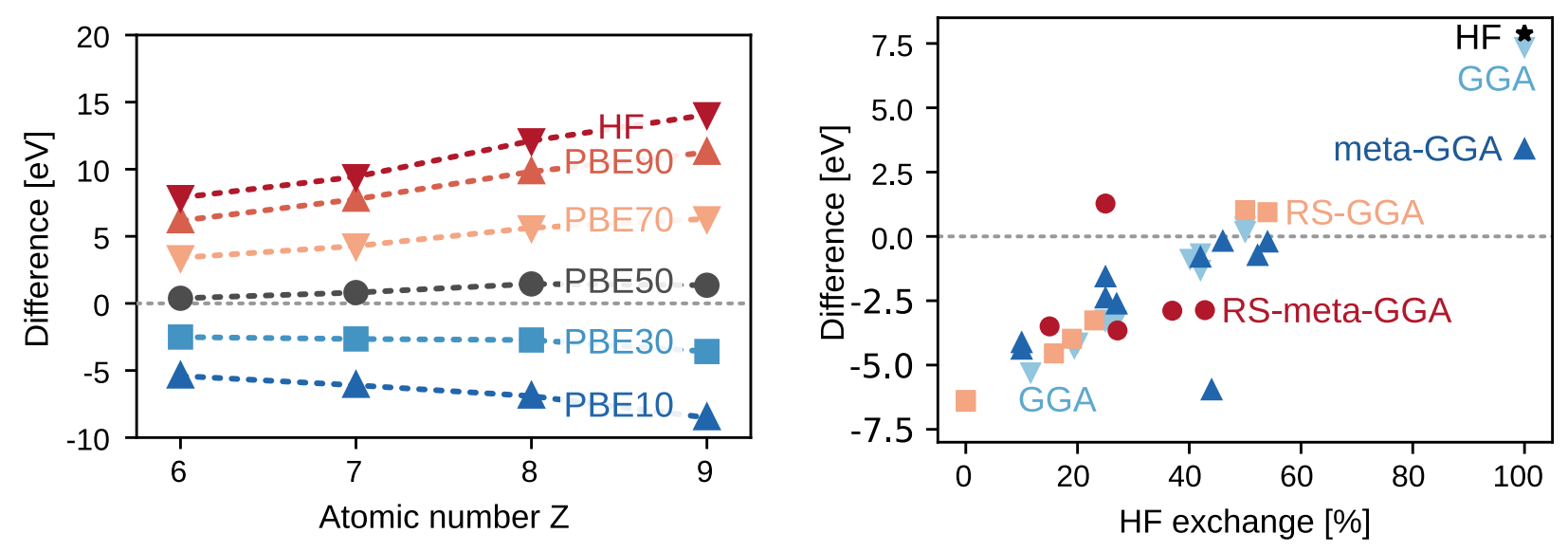

FIG. 3. Left: IE delocalization error as a function of atomic number, obtained using the $\triangle \mathrm{SCF}$ approach. DFT results obtained using the PBE functional with different amounts of HF exchange. Right: IE delocalization error of ethene, shown as a function of the amount of (short-range) HF exchange. Results obtained using hybrid GGA (light blue), meta-GGA (dark blue), range-separated GGA (orange), and range-separated meta-GGA (red) xc-functionals, with HF result shown as a black star.

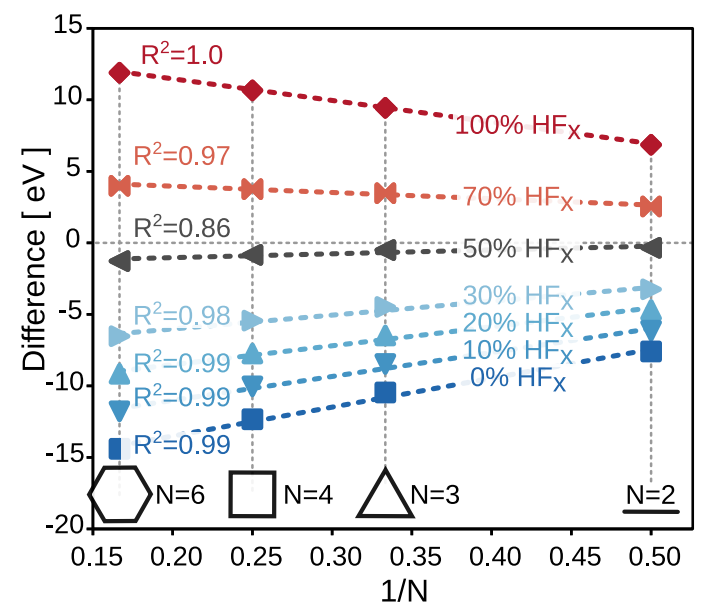

FIG. 4. IE delocalization error as a function of $1 / N$, where $N$ is the number of sites over which the core-hole is delocalized. The delocalized core-hole corresponds to the fully delocalized $\mathrm{C} 1 \mathrm{~s}$ bonding orbital in cycloalkanes of increasing size from ethene to cyclohexane. Differences obtained using various amounts of HF exchange included in a PBE-based functional are shown in different colors. The dotted lines are obtained by linear regression, with corresponding coefficients of determination.

$\left(E_{\mathrm{K}}\right)$, and DFT exchange and correlation $\left(E_{\mathrm{DFT}}\right)$ :

$$
\begin{aligned}
\mathrm{IE}= & \left(E_{\mathrm{H}}^{\mathrm{CH}}-E_{\mathrm{H}}^{\mathrm{GS}}\right)+\left(E_{\mathrm{J}}^{\mathrm{CH}}-E_{\mathrm{J}}^{\mathrm{GS}}\right)+\left(E_{\mathrm{K}}^{\mathrm{CH}}-E_{\mathrm{K}}^{\mathrm{GS}}\right) \\
& +\left(E_{\mathrm{DFT}}^{\mathrm{CH}}-E_{\mathrm{DFT}}^{\mathrm{GS}}\right),
\end{aligned}
$$

where individual components are defined as:

$$
\begin{aligned}
E_{\mathrm{H}} & =\sum_{i}\langle i|\hat{h}| i\rangle, \\
E_{\mathrm{J}} & =\frac{1}{2} \sum_{i, j}\langle i j \mid i j\rangle, \\
E_{\mathrm{K}} & =-\alpha \frac{1}{2} \sum_{i, j}\langle i j \mid j i\rangle, \\
E_{\mathrm{DFT}} & =(1-\alpha) \int f(\mathbf{n}(\mathbf{r}), \nabla \mathbf{n}(\mathbf{r})) \mathrm{d} \mathbf{r} .
\end{aligned}
$$

Here $i, j$ are occupied orbital indices, $|i\rangle$ are spin-orbitals, $\hat{h}$ is the Core-Hamiltonian operator, and the summation is over all occupied orbitals. The amount of HF exchange is given as $\alpha$, $f$ is a generic GGA functional of the electron density $(\mathbf{n})$, and its gradient $(\nabla \mathbf{n})$. The electron density, $\mathbf{n}(\mathbf{r})$, is obtained by representing the density matrix $\left(\mathbf{P}=\left\{P_{\mu \nu}\right\}\right)$ on a grid:

$$
P_{\mu \nu}=\sum_{i} C_{\mu i} C_{v i}
$$

where $\left\{C_{\mu i}\right\}$ are the $\mathrm{MO}$ coefficients. By restricting the summations in Eqs. (3)-(7) to core orbitals, core-electron contributions can be separated out:

$$
\begin{aligned}
& E_{\mathrm{H}}^{\mathrm{core}}=\sum_{I}\langle I|\hat{h}| I\rangle, \\
& E_{\mathrm{J}}^{\mathrm{core}}=\sum_{I, j}\langle I j \mid I j\rangle, \\
& E_{\mathrm{K}}^{\mathrm{core}}=-\alpha \sum_{I, j}\langle I j \mid j I\rangle .
\end{aligned}
$$

where $I$ are occupied core orbitals, and $j$ are generic occupied orbitals (including core). In the case of DFT exchangecorrelation, the core orbital contribution can be computed by determining the electron density required in Eq. (6) based on a density matrix constructed using only core orbitals:

$$
P_{\mu v}^{\mathrm{core}}=\sum_{I} C_{\mu I} C_{v I}
$$


For the exchange energy, we also calculated the core-core contribution of the $\beta$ spin channel alone (where $\beta$ is the spin channel with the core-hole):

$$
E_{\mathrm{K}}^{\mathrm{core}}=-\alpha \sum_{I, J}\left\langle I_{\beta} J_{\beta} \mid J_{\beta} I_{\beta}\right\rangle .
$$

In order to determine these terms for a localized and delocalized CH, localization using ECP:s will not be useful, as this will yield very different total energies and number of electrons. Instead, we localize the $\mathrm{CH}$ by using an asymmetric ethene molecule where one of the $\mathrm{CH}_{2}$ groups has $\mathrm{CH}$ bonds stretched by $0.05 \AA$ from the equilibrium value. This distortion is large enough to lift the degeneracy of the core MOs, allowing full localization on the atomic sites, but small enough not to affect the IE:s significantly. With this, energy differences $(\Delta E)$ of the contributions defined in Eqs. (3)-(6), (8)(10), and (12) are determined as:

$$
\Delta E=\left(E_{\mathrm{deloc}}^{\mathrm{CH}}-E_{\mathrm{deloc}}^{\mathrm{GS}}\right)-\left(E_{\mathrm{loc}}^{\mathrm{CH}}-E_{\mathrm{loc}}^{\mathrm{GS}}\right),
$$

The resulting breakdown in contributions is illustrated in Fig. 5, as a function of the amount of HF exchange mixed in a hybrid PBE-based xc-functional. The left panel shows total components (full lines), as well as those obtained using only the $\mathrm{C} 1 \mathrm{~s}$ core orbitals (dotted lines). All contributions display a close to linear dependence with respect to the amount of $\mathrm{HF}$ exchange, and we see that the main variation in IE delocalization error is due to the variation in HF exchange and DFT exchange-correlation. The difference in one-electron energy is quite small, and the Coulomb energy contributes mainly by a shift. Importantly, the large variations observed for HF exchange and DFT exchange-correlation stem mainly from the core orbitals, as the corresponding dotted lines lie almost perfectly over the total variations. The difference in Coulomb is also primarily involving the core, although a larger contribution from the other MOs is present there.

The right panel of Fig. 5 shows the variation in core Coulomb energy and core DFT exchange-correlation, alongside the HF exchange contribution of the core $\beta$ spin channel (the channel with the $\mathrm{CH}$ ). It is evident that the behaviour of the delocalization error stems almost entirely in the core $\beta$ channel. Furthermore, if these three energy components are summed together (black dotted line), the trend in IE error is almost perfectly captured, demonstrating that the error behaviour essentially originates due to the inadequate treatment of electron exchange and correlation for the core orbitals, specifically in the spin channel where the $\mathrm{CH}$ resides. As such, for high fractions of HF exchange the smaller core $\beta$ exchange in the final state when using a more diffuse $\mathrm{CH}$ dominates, leading to positive delocalization errors for fractions above about 0.5 . For smaller fractions the less influential core DFT exchange-correlation energy cannot compensate for the decreasing HF exchange energy, and the Coulomb term starts dominating, resulting in delocalization errors smaller than zero. The stronger core Coulomb contribution for the localized solution is largely due to the effective charge of the nuclei changing by one or one-half for the localized and delocalized picture, respectively, leading to more substantial density contractions and resulting Coulomb interactions.

\section{Post-Hartree-Fock methods and the influence of electron correlation}

To investigate the influence of electron correlation on the delocalization error, Fig. 6 reports XPS spectra for ethene at various post-HF levels of theory, as obtained from total energy differences. With a localized $\mathrm{CH}$, the resulting IE:s are all close to the experimental value of $290.7 \mathrm{eV},{ }^{97}$ with $\operatorname{CCSD}(\mathrm{T})$ yielding an IE of $290.9 \mathrm{eV}$ (accounting for a relativistic shift of about $0.1 \mathrm{eV}$ ). Using a delocalized $\mathrm{CH}$, much larger deviations are visible, with $\mathrm{HF}$ results being about $8 \mathrm{eV}$ too high in energy. As previously discussed, this is due to a lack of relaxation (and, to a smaller extent, correlation), and the agreement improves significantly once electron correlation is included. Even so, the variations remains noticeable, with a difference of $0.5 \mathrm{eV}$ remaining at the $\operatorname{CCSD}(\mathrm{T})$ level of theory. As such, relatively high order in perturbation theory is needed to yield identical results, and we note that this increases the likelihood of numerical instabilities due to the use of a non-Aufbau reference state. $^{73}$

In order to better understand the influence of electron correlation, Fig. 7 shows a breakdown of the MP2 energy corrections for a localized and delocalized $\mathrm{CH}$. We again use symmetric and asymmetric structures of ethene, and thus note that there will be a small error due to this use of different structures. The total MP2 energy correction is the sum of the same-spin $\left(E_{\mathrm{SS}}\right)$ and opposite-spin $\left(E_{\mathrm{OS}}\right)$ terms, which are calculated as:

$$
\begin{aligned}
E_{\mathrm{SS}} & =-\sum_{i, j, a, b, \sigma} \frac{\left\langle i_{\sigma} j_{\sigma} \mid a_{\sigma} b_{\sigma}\right\rangle\left\langle i_{\sigma} j_{\sigma}|| a_{\sigma} b_{\sigma}\right\rangle}{\varepsilon_{a}+\varepsilon_{b}-\varepsilon_{i}-\varepsilon_{j}}, \\
E_{\mathrm{OS}} & =-\sum_{i, j, a, b, \sigma, \sigma^{\prime}} \frac{\left\langle i_{\sigma} j_{\sigma^{\prime}} \mid a_{\sigma} b_{\sigma^{\prime}}\right\rangle\left\langle i_{\sigma} j_{\sigma^{\prime}} \mid a_{\sigma} b_{\sigma^{\prime}}\right\rangle}{\varepsilon_{a}+\varepsilon_{b}-\varepsilon_{i}-\varepsilon_{j}} .
\end{aligned}
$$

Here $\sigma$ indicates the spin, $i$ and $j$ denote occupied orbitals, and $a$ and $b$ virtual orbitals. By restricting these summations over different occupied spaces, the total MP2 correlation energy can be divided into separate components involving only valence-occupied orbitals, only core-occupied orbitals, and mixed core- and valence-occupied orbitals. This can also be done using frozen core or valence orbitals, with core-valence contribution being the remaining MP2 energy of the full-space calculation. ${ }^{98}$

As seen in Fig. 7, the largest difference lies in the corevalence correlation contribution of the delocalized $\mathrm{CH}$, which is about $9 \mathrm{eV}$ larger than the near-zero core-valence contributions of the ground state and localized $\mathrm{CH}$. Looking more closely at the precise terms, this discrepancy is dominated by same-spin $\beta$ and the opposite-spin contribution involving a core- $\beta$ orbital. Returning to the results presented and discussed in connection to Fig. 5, we see that the inconsistent exchange energy which is achieved when creating a delocalized $\mathrm{CH}$ is primarily accounted for by correlating the remaining $\beta$ orbitals with the valence region, although we note that the difference in $\triangle \mathrm{MP} 2 \mathrm{IE}$ remains relatively large at around $2 \mathrm{eV}$. Small additional differences are seen in the valence-valence and core-core contributions, where in particular the valencevalence contribution to the delocalized $\mathrm{CH}$ is shifted by about 

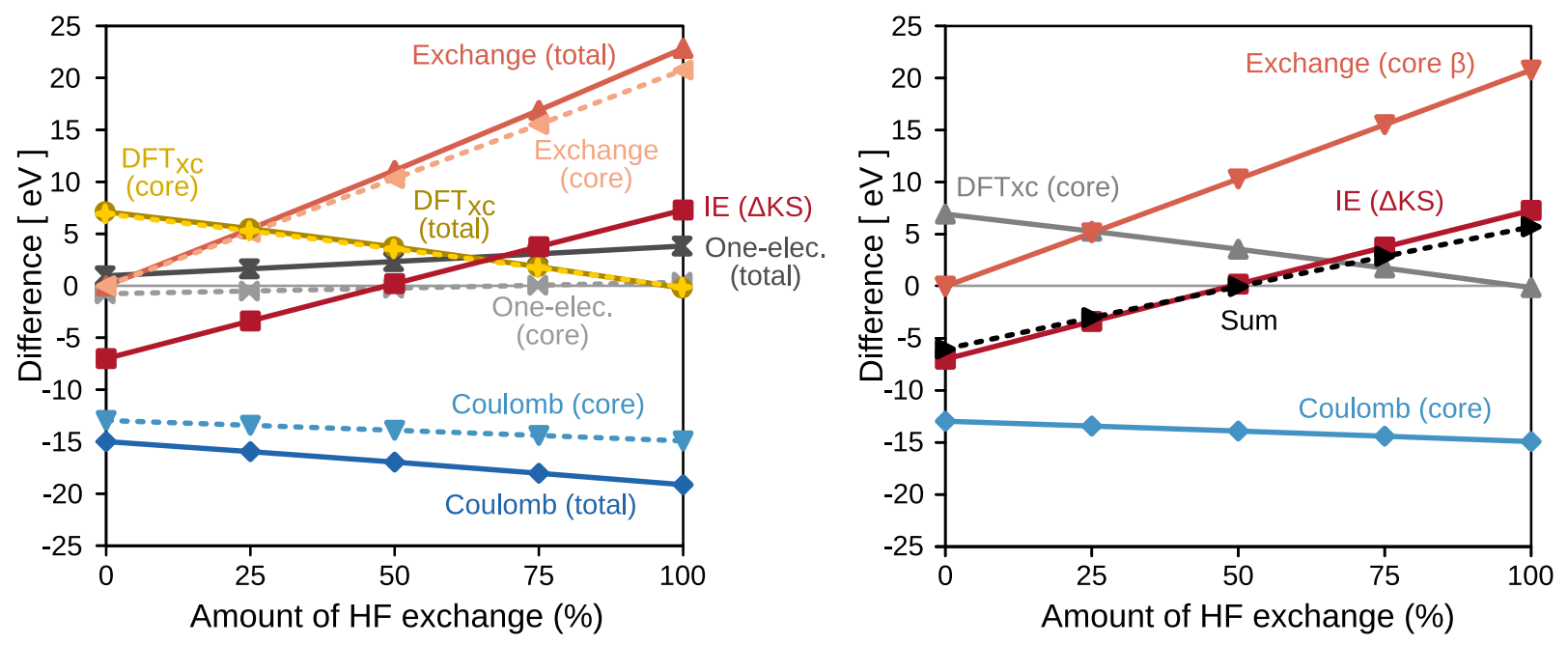

FIG. 5. Difference in IE energy terms using a localized or delocalized core-hole on ethene, as a function of the amounts of HF exchange included in a PBE-based hybrid functional. Left: IE difference decomposed into: one-electron contribution (gray), Coulomb (blue), HF exchange (pink), and DFT exchange and correlation (yellow). The contributions to these energy differences from only the core electrons are also shown (dotted lines). Right: the IE difference and the main contributions: the HF exchange contribution from the core $\beta$ electrons (spin channel with a $\mathrm{CH}$ ), DFT exchange and correlation of the core levels, and the Coulomb interaction for the core levels.

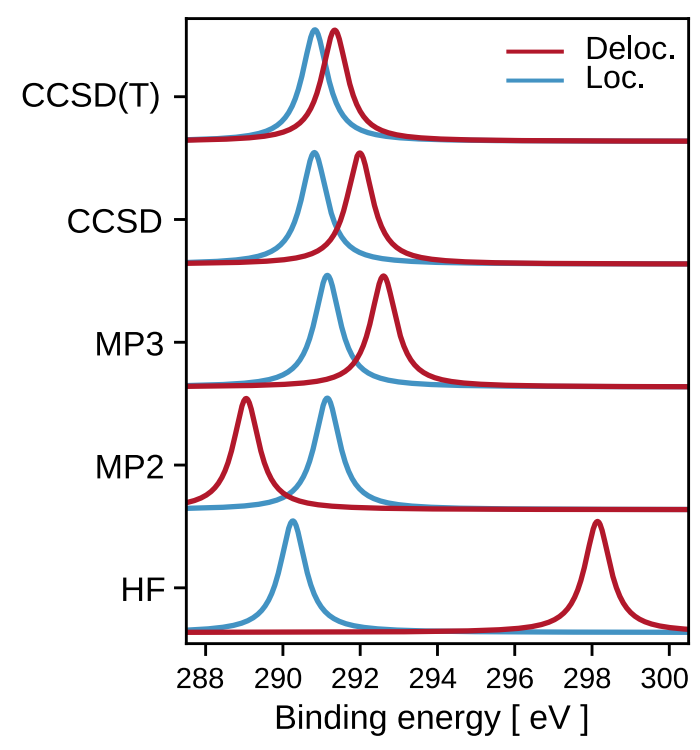

FIG. 6. X-ray photoelectron spectra of ethene calculated at different levels of theory, as obtained using a delocalized (red) and localized (blue) core-hole.

$0.8 \mathrm{eV}$. The core-core contribution of the neutral system is about $2 \mathrm{eV}$, which is lowered to $1 \mathrm{eV}$ for a localized $\mathrm{CH}$, or $1.5 \mathrm{eV}$ for a delocalized $\mathrm{CH}$. For a localized $\mathrm{CH}$, this decrease by one-half is easy to understand, as the same-spin contribution is close to zero due to the near-zero spatial overlap, and one out of two (close to equal) opposite-spin contribution disappears when a core-hole is created.

In terms of X-ray emission spectrum calculations, the spectra obtained with a localized and delocalized $\mathrm{CH}$ at different levels of theory are reported in Fig. 8. As seen above, XES is the spectroscopy most affected by $\mathrm{CH}$ localization discussed here, with both absolute energies and relative energies and intensities being significantly affected. We here see that ADC(1) shows particularly poor comparison between the two $\mathrm{CH}$ localization schemes, as this method lacks in both relaxation and correlation. For higher-order ADC and EOM-CCSD, the absolute energy discrepancy is much smaller $(0.3-4.1 \mathrm{eV}$, with either sign), and the relative features are in better agreement. Still, the difference remains large, and we recommend using the localized $\mathrm{CH}$ approach for practical calculations.

\section{E. Size of the CVS space}

A different context in which core MO localization becomes important is in the selection of a CVS space for calculating Xray absorption spectra. Generally, the core-valence separation is justified from the observation that the core- and valenceorbitals are well-separated both spatially and energetically. Still, there are cases where a CVS space containing only some of the core MOs and leaving out others of the same atomic type may be useful. For example, such a tailored CVS space can be used to lower computational cost, as well as to avoid state mixing which can make analysis more difficult. ${ }^{27,28}$ Doing so breaks the energetic argument in CVS, so this tailoring relies on the coupling between orbitals still being small from a spatial perspective. This is clearly not the case if MOs delocalized over different atomic sites are set in separated CVS spaces, as will now be discussed.

Fig. 9 shows the X-ray absorption spectrum of ethene, as obtained when using a CVS space consisting of both core MOs, or tailored spaces containing one MO at a time. Furthermore, results obtained when localizing the core orbitals with ECP:s are also shown, yielding practically identical re- 


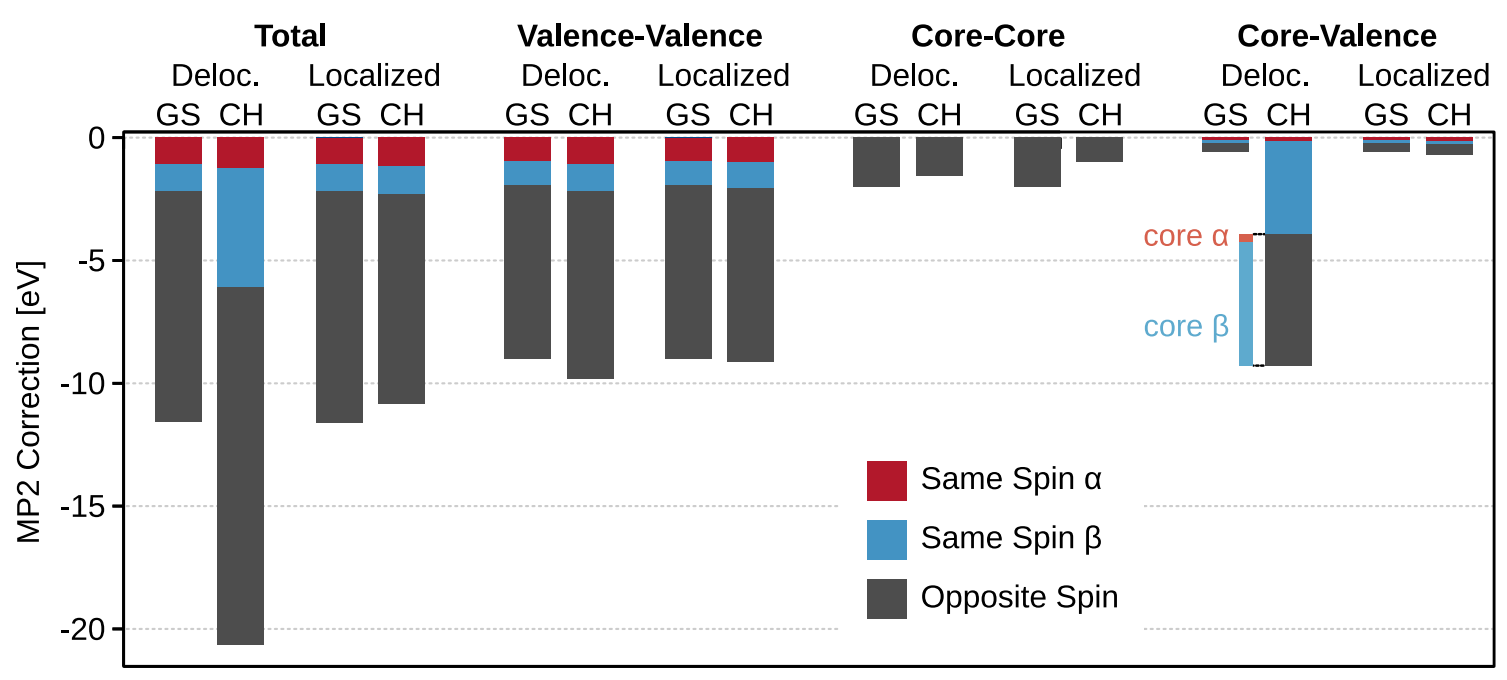

FIG. 7. MP2 energy corrections for the ground state (GS) and core-hole (CH) state of symmetric (delocalized $\mathrm{CH}$ ) and asymmetric (localized $\mathrm{CH})$ ethene. The energy corrections have been decomposed into different contributions, categorized by the involved MO levels and spin components. The opposite spin MP2 correction of the core-valence term has been further divided into contributions involving core $\alpha$ and the remaining core $\beta$ orbitals ( $\mathrm{CH}$ places in $\beta$ ).

sults as when using the full CVS space. It is clear that using a CVS space tailored to each core $\mathrm{MO}$ at a time introduces an error in both absolute and relative energies and features. For $\mathrm{ADC}(1)$, the difference in absolute energy is the smallest, as neither the full nor the tailored calculations include relaxation. By comparison, the relative features are quite different. The closest agreement in relative features is present for $\operatorname{ADC}(2)-x$, which also has the largest absolute energy difference, as well as being the method which has been shown to perform best for XAS. ${ }^{28,69}$

Moving to the more interesting case of non-equivalent atomic sites, the use of a full or tailored CVS spaces for cyclopentadiene and furan is illustrated in Fig. 10. Here, the tailored spectra are constructed by considering separately each set of (what can be thought to be) MOs of chemically unique atoms. For furan, the tailored CVS approach works very well, while for cyclopentadiene this approach clearly does not work. This is because the $\mathrm{C}_{1}$ and $\mathrm{C}_{2}$ sets of chemically nonequivalent atoms in cyclopentadiene have closer $\mathrm{C}$ $1 \mathrm{~s}$ binding energies, with the corresponding MOs partially delocalized over all atomic sites.

As such, in order to reduce the computational costs by considering separate CVS spaces, delocalization over different sets of symmetry-unique atoms needs to be avoided. This can be ensured by checking the delocalization of the ground state MOs, or by using ECP:s and considering each different site at a time. While using a full CVS space is the approach most consistent with the original formulation of the core-valence separation approximation, the use of tailored CVS spaces can lower computational cost by reducing matrix sizes, as well as the number of eigenstates needed at a time. The approach thus has benefits, but some care must be observed.

\section{CONCLUSIONS}

In summary, we have analyzed the influence of core-hole $(\mathrm{CH})$ delocalization on X-ray photoionization, X-ray absorption and X-ray emission spectra calculated at various levels of theory, including TP-DFT, TDDFT, and the post-HF correlated methods ADC and CC. In cases where an explicit $\mathrm{CH}$ is required in the calculation of a highly symmetric molecule with chemically equivalent atoms, namely for IE/XPS and XAS (TP-DFT), or XES (TDDFT, TP-DFT, ADC, CC), the use of a delocalized core-hole can produce a large error. In the case of IE/XPS and XAS, the error manifests mainly in terms of an absolute energy shift. By comparison, XES calculations are sensitive both in terms of absolute energies, as well as for relative peak positions and intensities. The size of the $\mathrm{CH}$ delocalization error depends on the element (the larger the atomic number, the larger the error), on the number of sites over which the CG is delocalized (the more sites, the larger the error), as well as on the description of exchange and correlation. The source of the errors are traced back to the inappropriate treatment of electron correlation and orbital relaxation, ${ }^{1-3}$ where the progressive improvement of correlation treatment results in the gradual reduction of the error. In the case of DFT, the error is directly related to the amount of HF exchange included in the xc-functional, where the improper description of the exchange interaction between the core orbitals with the same spin as the delocalized core-hole constitutes the predominant contribution.

As a best practice, for state-specific calculations which require an explicit core-hole, the recommendation is to use a localized core-hole, especially if the system under investigation has a high degree of symmetry. This can be done by, e.g., using ECP:s for all atoms of the same type save the probed one. Moreover, care must be given to calculations which involve tailored CVS spaces. In this case, all nearly degenerate 

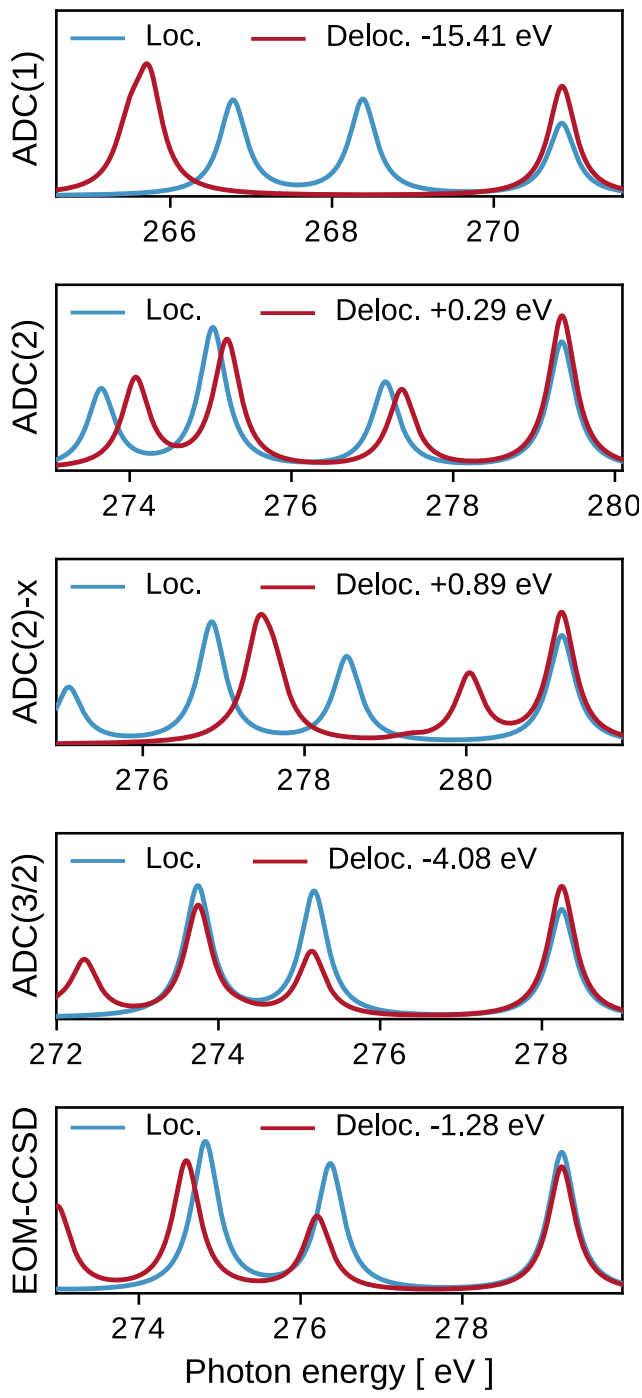

FIG. 8. X-ray emission spectra of ethene calculated with a localized (blue) and delocalized (red) core-hole, as obtained using EOMCCSD and with the ADC hierarchy. Delocalized $\mathrm{CH}$ results are shifted in energy such that the high-energy features overlap.

core orbitals must be included in the same CVS space to avoid errors, or ECP:s can again be used.

\section{ACKNOWLEDGEMENTS}

T.F. acknowledges the financial support of the Air Force Office of Scientific Research (Grant FA8655-20-1-7010). I.E.B. acknowledges support provided by Prof. Y.M. Rhee through a grant from the National Research Foundation (NRF) of Korea (No. 2020R1A5A1019141) funded by the Ministry of Science and ICT (MSIT). I.E.B also acknowledges support from the Swedish Research Council (Grant 2017-06419). We thank Patrick Norman for constructive feedback on our manuscript. The calculations were enabled by resources provided by the Swedish National Infrastructure for Computing
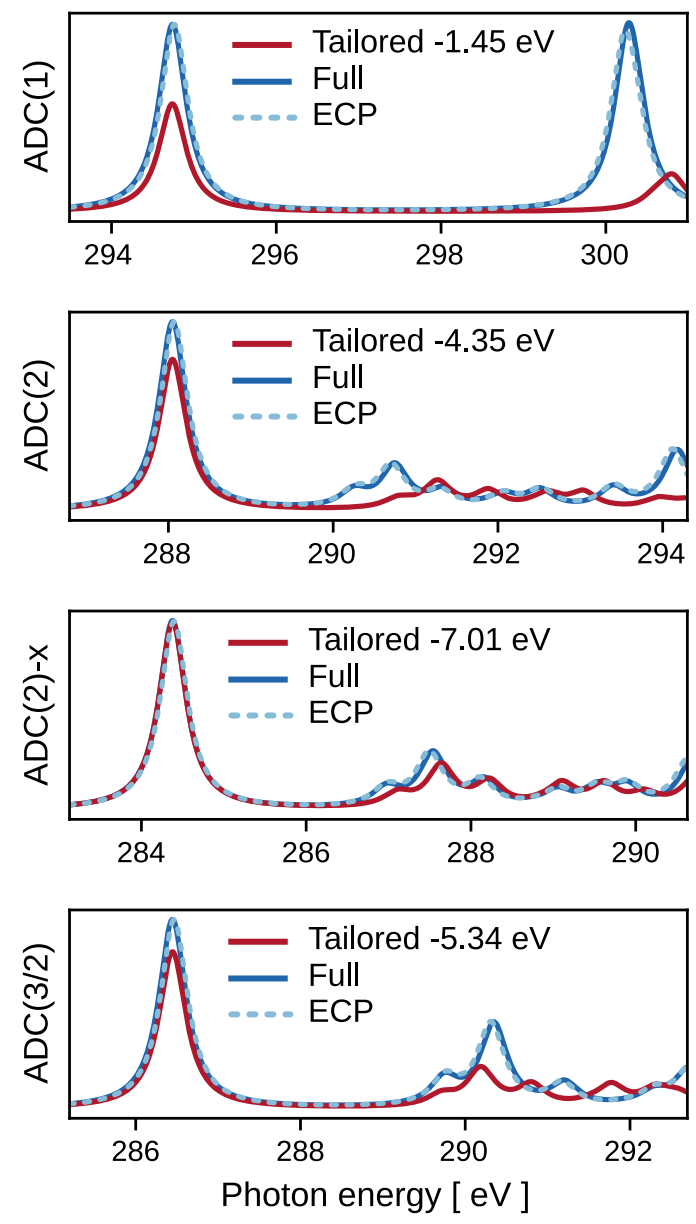

FIG. 9. X-ray absorption spectrum of ethene calculated using several orders of CVS-ADC with a full CVS space (blue), ECP:s (blue dotted), or by considering the two C 1s core MOs in separate CVS spaces (red). The tailored CVS spectrum is the sum of these two individual contributions and has been shifted in energy to overlap with the first feature of the full CVS calculation.

(SNIC) at NSC, partially funded by the Swedish Research Council through grant agreement No. 2018-05973.

\section{DATA AVAILABILITY STATEMENT}

The data that support the findings of this study are available from the corresponding author upon reasonable request.

\section{REFERENCES}

${ }^{1}$ P. S. Bagus and H. F. Schaefer, "Localized and delocalized 1s hole states of the $\mathrm{O}_{2}^{+}$molecular ion," J. Chem. Phys. 56, 224 (1972).

${ }^{2}$ B. T. Pickup and O. Goscinski, "Direct calculation of ionization energies," Mol. Phys. 26, 1013 (1973).

${ }^{3}$ L. S. Cederbaum and W. Domcke, "Localized and delocalized core holes and their interrelation,’ J. Chem. Phys. 66, 5084 (1977). 

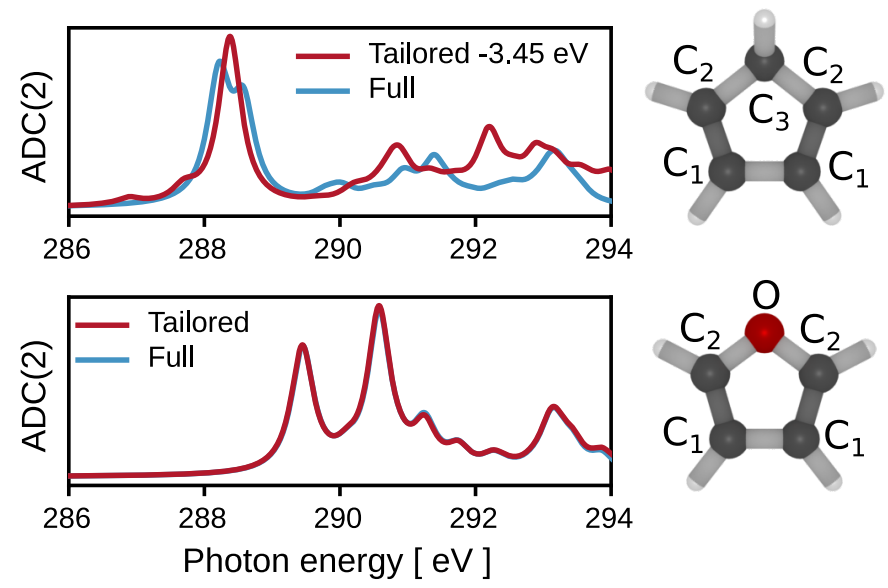

FIG. 10. X-ray absorption spectra of cyclopentadiene (top) and furan (bottom) calculated at the CVS-ADC(2) level of theory. Results obtained using the full CVS space encompassing all carbon atoms (blue), or tailored CVS spaces including the chemically nonequivalent $\mathrm{C}$ atoms separately (red). The tailored CVS spectrum of cyclopentadiene has been shifted such that the peak max approximately overlaps with that of the full CVS calculation.

${ }^{4}$ A. B. Rocha and C. E. V. de Moura, "The problem of hole localization in inner-shell states of $\mathrm{N}_{2}$ and $\mathrm{CO}_{2}$ revisited with complete active space selfconsistent field approach," J. Chem. Phys. 135, 224112 (2011).

${ }^{5}$ H. Ågren, P. S. Bagus, and B. O. Roos, "Symmetry adapted versus symmetry broken wavefunctions: The 1 s core level ions of $\mathrm{O}_{2}^{+}$," Chem. Phys. Lett. 82, 505 (1981).

${ }^{6}$ W. H. E. Schwarz, T. C. Chang, U. Seeger, and K. H. Hwang, "Core excitations of symmetrical aromatic molecules. specific correlations in the valence shell and localization in the core shell," Chem. Phys. 117, 73 (1987).

${ }^{7}$ M. S. Schöffler, J. Titze, N. Petridis, T. Jahnke, K. Cole, L. P. H. Schmidt, A. Czasch, D. Akoury, O. Jagutzki, and J. B. Williams et al., "Ultrafast probing of core hole localization in $\mathrm{N}_{2}$," Science 320, 920-923 (2008).

${ }^{8}$ O. Björneholm, M. M. Bässler, A. Ausmees, I. Hjelte, R. Feifel, H. Wang, C. Miron, M. N. Piancastelli, S. Svensson, and S. L. Sorensen et al., "Doppler splitting of in-flight Auger decay of dissociating oxygen molecules: The localization of delocalized core holes," Phys. Rev. Lett. 84, 2826 (2000).

${ }^{9}$ R. Guillemin, P. Decleva, M. Stener, C. Bomme, T. Marin, L. Journel, T. Marchenko, R. K. Kushawaha, K. Jänkälä, and N. Trcera et al., "Selecting core-hole localization or delocalization in $\mathrm{CS}_{2}$ by photofragmentation dynamics," Nat. Comm. 6, 6166 (2020).

${ }^{10}$ H. Sann, C. Schober, A. Mhamdi, F. Trinter, C. Müller, S. Semenov, M. Stener, M. Waitz, T. Bauer, and R. Wallauer et al., "Delocalization of a vacancy across two neon atoms bound by the van der Waals force," Phys. Rev. Lett. 117, 263001 (2016).

${ }^{11}$ V. Ekholm and et al., "Core-hole localization and ultra-fast dissociation in $\mathrm{SF}_{6}$," J. Phys. B: At. Mol. Opt. Phys. 53, 185101 (2020).

${ }^{12}$ J. Müller, H. Agren, and O. Goscinski, "Role of localization in the predition of core ESCA lineshapes of $\mathrm{N}_{2}, \mathrm{O}_{2}$ and NO," Chem. Phys. 38, 349 (1979).

${ }^{13}$ L. C. Snyder, "Core-electron binding energies and slater atomic shielding constants," J. Chem. Phys. 55, 95 (1971).

${ }^{14} \mathrm{~S}$. Canuto, O. Goscinski, and M. Zerner, "Broken orbital symmetry study of low-lying excited and N1s ionized states of pyrazine," Chem. Phys. Lett. 68, 232 (1979).

${ }^{15} \mathrm{~F}$. Gel'mukhanov and H. Ågren, "Measurements of core hole localization in X-ray raman scattering," JETP Lett. 67, 1064 (1998).

${ }^{16}$ S. A. Malinovskaya and L. S. Cederbaum, "Violation of electronic optical selection rules in X-ray emission by nuclear dynamics: Time-dependent formulation,” Phys. Rev. A 61, 042706 (2000).

${ }^{17}$ J. A. Kinstop and W. V. M. Machado, "Hole localization versus delocalization in symmetric molecular ions," Phys. Rev. A 43, 3348 (1991).
${ }^{18}$ N. V. Kryzhevoi, N. V. Dobrodey, and L. S. Cederbaum, "Core-hole Hamiltonians and corrected equivalent core model for systems with equivalent atoms," J. Chem. Phys. 119, 12138 (2003).

${ }^{19}$ C. A. Marante, L. Greenman, C. S. Trevisan, T. N. Rescigno, C. W. McCurdy, and R. R. Lucchese, "Validity of the static-exchange approximation for inner-shell photoionization of polyatomic molecules," Phys. Rev. A 102, 012815 (2020).

${ }^{20}$ L. S. Cederbaum, F. Tarantelli, A. Sgamellotti, and J. Schirmer, "On double vacancies in the core," J. Chem. Phys. 85, 6513 (1986).

${ }^{21}$ M. Tashiro, M. Ehara, and K. Ueda, "Double core-hole electron spectroscopy for open-shell molecules: Theoretical perspective," Chem. Phys. Lett. 496, 217-222 (2010).

${ }^{22}$ J. Lee, D. W. Small, and M. Head-Gordon, "Excited states via coupled cluster theory without equation-of-motion methods: Seeking higher roots with application to doubly excited states and double core hole states," J. Chem. Phys 151, 214103 (2019).

${ }^{23}$ Y. Zhang, U. Bergmann, R. Schoenlein, M. Khalil, and N. Govind, "Double core hole valence-to-core X-ray emission spectroscopy: A theoretical exploration using time-dependent density functional theory," J. Chem. Phys. 151, 144114 (2019).

${ }^{24} \mathrm{~V}$. Carravetta and H. Ågren, "Symmetry breaking and hole localization in multiple core electron ionization," J. Phys. Chem. A 117, 6798 (2013).

${ }^{25}$ C. J. Clarke, S. Hayama, A. Hawes, J. P. Hallett, T. W. Chamberlain, K. R. J. Lovelock, and N. A. Besley, "Zinc 1s valence-to-core X-ray emission spectroscopy of halozincate complexes," J. Phys. Chem. A 123, 9552-9559 (2019).

${ }^{26} \mathrm{D}$. Hait and M. Head-Gordon, "Highly accurate prediction of core spectra of molecules at density functional theory cost: Attaining sub-electronvolt error from a restricted open-shell Kohn-Sham approach,” J. Phys. Chem. Lett. 11, 775-786 (2020).

${ }^{27}$ M. F. Herbst, M. Scheurer, T. Fransson, D. R. Rehn, and A. Dreuw, "adcc: A versatile toolkit for rapid development of algebraic-diagrammatic construction methods," WIREs Comput. Mol. Sci. e1462 (2020).

${ }^{28}$ T. Fransson, I. E. Brumboiu, M. L. Vidal, P. Norman, S. Coriani, and A. Dreuw, "XАBOOM: An X-ray absorption benchmark of organic molecules based on carbon, nitrogen, and oxygen $1 s \rightarrow \pi^{*}$ transitions," J. Chem. Theory Comput. 17, 1618 (2021).

${ }^{29} \mathrm{P}$. Norman and A. Dreuw, "Simulating X-ray spectroscopies and calculating core-excited states of molecules," Chem. Rev. 118, 7208-7248 (2018).

${ }^{30}$ P. S. Bagus and H. F. Schaefer, "Direct near-Hartree-Fock calculations of the 1s hole states of $\mathrm{NO}^{+}$, , J. Chem. Phys. 55, 1474 (1971).

${ }^{31}$ A. T. B. Gilbert, N. A. Besley, and P. M. W. Gill, "Self-consistent field calculations of excited states using the maximum overlap method (MOM)," J. Phys. Chem. A 112, 13164-13171 (2008).

${ }^{32}$ G. M. J. Barca, A. T. B. Gilbert, and P. M. W. Gill, "Simple models for difficult electronic excitations," J. Chem. Theory Comput. 14, 1501 (2018).

${ }^{33}$ K. Carter-Fenk and J. M. Herbert, "State-targeted energy projection: A simple and robust approach to orbital relaxation of non-Aufbau self-consistent field solutions," J. Chem. Theory Comput. 16, 5067 (2020).

${ }^{34}$ D. Hait and M. Head-Gordon, "Excited state orbital optimization via minimizing the square of the gradient: General approach and application to singly and doubly excited states via density functional theory," J. Chem. Theory Comput. 16, 1699 (2020).

${ }^{35}$ J. C. Slater, "Statistical exchange-correlation in the self-consistent field," Adv. Quant. Chem. 6, 1 (1972).

${ }^{36}$ J. C. Slater and K. H. Johnsson, "Self-consistent-field X $\alpha$ cluster method for polyatomic molecules and solids," Phys. Rev. B 5, 844 (1972).

${ }^{37}$ M. Stener, A. Lisini, and P. Decleva, "Density functional calculations of excitation energies and oscillator strengths for $\mathrm{C} 1 \mathrm{~s} \rightarrow \pi^{*}$ and $\mathrm{O} 1 \mathrm{~s} \rightarrow \pi^{*}$ excitations and ionization potentials in carbonyl containing molecules," Chem. Phys. 191, 141 (1995).

${ }^{38}$ L. Triguero, L. G. M. Pettersson, and H. Ågren, "Calculations of nearedge X-ray-absorption spectra of gas-phase and chemisorbed molecules by means of density-functional and transition-potential theory," Phys. Rev. B 58, 8097-8110 (1998).

${ }^{39}$ M. Nyberg, Y. Luo, L. Triguero, L. G. M. Pettersson, and H. Ågren, "Corehole effects in x-ray-absorption spectra of fullerenes," Phys. Rev. B 60, 7956-7960 (1999).

${ }^{40}$ D. Prendergast and G. Galli, "X-ray absorption spectra of water from first principles calculations," Phys. Rev. Lett. 96, 21 (2006). 
${ }^{41}$ C. Kolczewski, R. Püttner, M. Martins, A. S. Schlachter, G. Snell, M. M. Sant'Anna, K. Hermann, and G. Kaindl, "Spectroscopic analysis of small organic molecules: A comprehensive near-edge X-ray-absorption finestructure study of $\mathrm{C}_{6}$-ring-containing molecules," J. Chem. Phys. 124, 034302 (2006).

${ }^{42}$ M. Simons and D. A. Matthews, "Transition-potential coupled cluster," J. Chem. Phys. 154, 014106 (2020).

${ }^{43}$ N. Lee, T. Petrenko, U. Bergmann, U. Neese, and S. DeBeer, "Probing valence orbital composition with iron $\mathrm{K} \beta \mathrm{X}$-ray emission spectroscopy," J. Am. Chem. Soc. 132, 9715-9727 (2010).

${ }^{44} \mathrm{~N}$. Besley, "Modeling of the spectroscopy of core electrons with density functional theory," WIREs Comput. Mol. Sci e1527 (2021).

${ }^{45}$ T. Zhang, I. E. Brumboiu, V. Lanzilotto, C. Grazioli, A. Guarnaccio, F. O. L. Johansson, M. Coreno, M. de Simone, A. Santagata, B. Brena, and C. Puglia, "Electronic structure modifications induced by increased molecular complexity: from triphenylamine to m-MTDATA," Phys. Chem. Chem. Phys. 21 (2019).

${ }^{46}$ D. Erbahar, T. Susi, X. Rocquefelte, C. Bittencourt, M. Scardamaglia, P. Blaha, P. Guttmann, G. Rotas, N. Tagmatarchis, X. Zhu, A. P. Hitchcock, and C. P. Ewels, "Spectromicroscopy of $\mathrm{C}_{60}$ and azafullerene $\mathrm{C}_{5} 9 \mathrm{~N}$ : Identifying surface adsorbed water," Sci. Rep. 6 (2016).

${ }^{47}$ I. E. Brumboiu, A. S. Anselmo, B. Brena, A. Dzwilewski, K. Svensson, and E. Moons, "Near-edge x-ray absorption fine structure study of the $c_{60^{-}}$ derivative pcbm," Chem. Phys. Lett. 568 (2013).

${ }^{48}$ E. Diesen, G. L. Rodrigues, A. C. Luntz, F. Abild-Pedersen, L. G. Pettersson, and J. Voss, "Accuracy of xas theory for unraveling structural changes of adsorbates: Co on ni (100)," AIP Advances 10, 115014 (2020).

${ }^{49}$ P. Jiang, D. Prendergast, F. Borondics, S. Porsgaard, L. Giovanetti, E. Pach, J. Newberg, H. Bluhm, F. Besenbacher, and M. Salmeron, "Experimental and theoretical investigation of the electronic structure of $\mathrm{Cu}_{2} \mathrm{O}$ and $\mathrm{CuO}$ thin films on $\mathrm{Cu}(110)$ using $\mathrm{X}$-ray photoelectron and absorption spectroscopy," J. Chem. Phys. 138, 024704 (2013).

${ }^{50}$ M. P. Ljungberg, J. J. Mortensen, and L. G. M. Pettersson, “An implementation of core level spectroscopies in a real space projector augmented wave density functional theory code," J. Electron Spectrosc. Relat. Phenom. 184, 427-439 (2011)

${ }^{51}$ P. Norman, "A perspective on nonresonant and resonant electronic response theory for time-dependent molecular properties," Phys. Chem. Chem. Phys. 13, 20519-20535 (2011).

${ }^{52}$ H. Ågren, V. Carravetta, O. Vahtras, and L. G. M. Pettersson, "Direct SCF direct static-exchange calculations of electronic spectra," Theor. Chem. Acc. 97, 14-40 (1997).

${ }^{53}$ A. Dreuw and M. Head-Gordon, "Single-reference ab initio methods for the calculation of excited states of large molecules," Chem. Rev. 105, 40094037 (2005).

${ }^{54}$ S. Hirata and M. Head-Gordon, "Time-dependent density functional theory within the Tamm-Dancoff approximation," Chem. Phys. Lett. 314, 291 (1999).

${ }^{55}$ J. P. Perdew and A. Zunger, "Self-interaction correction to densityfunctional approximations for many-electron systems," Phys. Rev. B 23, 5048-5079 (1981).

${ }^{56}$ N. A. Besley and F. A. Asmuruf, "Time-dependent density functional theory calculations of the spectroscopy of core electrons," Phys. Chem. Chem. Phys. 12, 12024-12039 (2010).

${ }^{57}$ Y. Imamura and H. Nakai, "Analysis of self-interaction correction for describing core excited states," Int. J. Quantum Chem. 107, 23-29 (2007).

${ }^{58} \mathrm{~N}$. A. Besley, "Density functional theory based methods for the calculation of X-ray spectroscopy," Acc. Chem. Res. 53, 1306 (2020).

${ }^{59}$ J. Schirmer, "Beyond the random-phase approximation: A new approximation scheme for the polarization propagator," Phys. Rev. A 26, 2395-2416 (1982).

${ }^{60} \mathrm{~A}$. Dreuw and M. Wormit, "The algebraic diagrammatic construction scheme for the polarization propagator for the calculation of excited states," WIREs Comput. Mol. Sci. 5, 82-95 (2015).

${ }^{61} \mathrm{~J}$. Schirmer, "Closed-form intermediate representations of many-body propagators and resolvent matrices," Phys. Rev. A 43, 4647 (1991).

${ }^{62}$ F. Mertins and J. Schirmer, "Algebraic propagator approaches and intermediate-state representations. I. The biorthogonal and unitary coupledcluster methods," Phys. Rev. A 53, 2140-2152 (1996).
${ }^{63}$ J. Schirmer and A. B. Trofimov, "Intermediate state representation approach to physical properties of electronically excited molecules," J. Chem. Phys. 120, 11449-11464 (2004).

${ }^{64}$ J. F. Stanton and R. J. Bartlett, "The equation of motion coupled-cluster method. A systematic biorthogonal approach to molecular excitation energies, transition probabilities, and excited state properties," J. Chem. Phys. 98, 7029 (1993)

${ }^{65}$ R. J. Bartlett, "Coupled-cluster theory and its equation-of-motion extensions," WIREs Comput. Mol. Sci. 2, 126 (2012).

${ }^{66} \mathrm{H}$. Koch and P. Jørgensen, “Coupled cluster response functions," J. Chem. Phys. 93, 3333 (1990).

${ }^{67}$ T. B. Pedersen and H. Koch, "Coupled cluster response functions revisited," J. Chem. Phys. 106, 8059 (1997).

${ }^{68} \mathrm{O}$. Christiansen, P. Jørgensen, and C. Hättig, "Response functions from fourier component variational perturbation theory applied to a timeaveraged quasienergy," Int. J. Quantum Chem. 68, 1-52 (1998).

${ }^{69}$ J. Wenzel, A. Holzer, M. Wormit, and A. Dreuw, "Analysis and comparison of CVS-ADC approaches up to third order for the calculation of coreexcited states," J. Chem. Phys. 142, 214104 (2015).

${ }^{70}$ M. L. Vidal, X. Feng, E. Epifanovsky, A. I. Krylov, and S. Coriani, "New and efficient equation-of-motion coupled-cluster framework for coreexcited and core-ionized states," J. Chem. Theory Comput. 15, 3117-3133 (2019).

${ }^{71}$ N. A. Besley, "Equation of motion coupled cluster theory calculations of the X-ray emission spectroscopy of water," Chem. Phys. Lett. 542, 42-46 (2012).

${ }^{72}$ T. Fransson and A. Dreuw, "Simulating X-ray emission spectroscopy with algebraic diagrammatic construction schemes for the polarization propagator," J. Chem. Theory Comput. 15, 546-556 (2019).

${ }^{73}$ A. Dreuw and T. Fransson, "Using core-hole reference states for calculating X-ray photoelectron and emission spectra," Submitted.

${ }^{74}$ L. S. Cederbaum, W. Domcke, and J. Schirmer, "Many-body theory of core holes," Phys. Rev. A 22, 206 (1980).

${ }^{75}$ J. Wenzel, M. Wormit, and A. Dreuw, "Calculating core-level excitations and X-ray absorption spectra of medium-sized closed-shell molecules with the algebraic-diagrammatic construction scheme for the polarization propagator," J. Comp. Chem. 35, 1900-1915 (2014).

${ }^{76}$ A. Barth and J. Schirmer, "Theoretical core-level excitation spectra of $\mathrm{N}_{2}$ and CO by a new polarisation propagator method," J. Phys. B: At. Mol. Phys. 18, 867-885 (1985).

${ }^{77}$ R. Peng, A. V. Copan, and A. Y. Sokolov, "Simulating X-ray absorption spectra with linear-response density cumulant theory," J. Phys. Chem. A 123, 1840-1850 (2019).

${ }^{78}$ M. Stener, G. Fronzoni, and M. de Simone, "Time dependent density functional theory of core electrons excitations," Chem. Phys. Lett. 373, 115-123 (2003).

${ }^{79}$ D. R. Rehn, A. Dreuw, and P. Norman, "Resonant inelastic X-ray scattering amplitudes and cross section in the algebraic diagrammatic construction/intermediate state representation (ADC/ISR) approach," J. Chem. Theory Comput. 13, 5552-5559 (2017).

${ }^{80}$ M. F. Herbst and T. Fransson, "Quantifying the error of the core-valence separation approximation," J. Chem. Phys. 153, 054114 (2020).

${ }^{81}$ J. D. Wadey and N. A. Besley, "Quantum chemical calculations of X-ray emission spectroscopy," J. Chem. Theory Comput. 10, 4557-4564 (2014).

${ }^{82}$ Y. Zhang, S. Mukamel, M. Khalil, and N. Govind, "Simulating valenceto-core X-ray emission spectroscopy of transition metal complexes with time-dependent density functional theory," J. Chem. Theory Comput. 11, 5804-5809 (2015).

${ }^{83} \mathrm{C}$. Møller and M. S. Plesset, "Note on an approximate treatment for manyelectron systems," Phys. Rev. 46, 618 (1934).

${ }^{84}$ T. H. Dunning, "Gaussian basis sets for use in correlated molecular calculations. I. The atoms boron through neon and hydrogen," J. Chem. Phys. 90, 1007-1023 (1989).

${ }^{85}$ E. Epifanovsky, A. T. B. Gilbert, X. Feng, J. Lee, Y. Mao, N. Mardirossian, P. Pokhilko, A. F. White, M. P. Coons, and A. L. Dempwolff et al., "Software for the frontiers of quantum chemistry: An overview of developments in the Q-Chem 5 package," J. Chem. Phys. 155, 084801 (2021).

${ }^{86}$ A. Bergner, M. Dolg, W. Küchle, H. Stoll, and H. Preuß, "Ab initio energyadjusted pseudopotentials for elements of groups 13-17," Mol. Phys. 80, 1431-1441 (1993). 
${ }^{87}$ J. P. Perdew, K. Burke, and M. Ernzerhof, "Generalized gradient approximation made simple," Phys. Rev. Lett. 77, 3865-3868 (1996).

${ }^{88}$ J. P. Perdew, K. Burke, and M. Ernzerhof, "Generalized gradient approximation made simple [Phys. Rev. Lett. 77, 3865 (1996)],’ Phys. Rev. Lett. 78, 1396-1396 (1997).

${ }^{89}$ A. Barth and L. S. Cederbaum, "Many-body theory of core-valence excitations," Phys. Rev. A 23, 1038 (1981).

${ }^{90}$ Q. Sun, T. C. Berkelbach, N. S. Blunt, G. H. Booth, S. Guo, Z. Li, J. Liu, J. D. McClain, E. R. Sayfutyarova, S. Sharma, S. Wouters, and G. K.L. Chan, "PySCF: the Python-based simulations of chemistry framework," WIREs Comput. Mol. Sci. 8, 1340 (2018)

${ }^{91}$ Q. Sun, X. Zhang, S. Banerjee, P. Bao, M. Barbry, N. S. Blunt, N. A. Bogdanov, G. H. Booth, J. Chen, Z.-H. Cui, and J. J. Eriksen et al., "Recent developments in the PySCF program package," J. Chem. Phys. 153, 024109 (2020).

${ }^{92}$ C. Ehlert and T. Klamroth, "PSIXAS: A Psi4 plugin for efficient simulations of X-ray absorption spectra based on the transition-potential and $\Delta$ Kohn-Sham method,” J. Comp. Chem 41, 1781 (2020).
${ }^{93}$ D. G. A. Smith, L. A. Burns, A. C. Simmonett, R. M. Parrish, M. C. Schieber, R. Galvelis, P. Kraus, H. Kruse, R. D. Remigio, and A. Alenaizan et al., "Psi4 1.4: Open-source software for high-throughput quantum chemistry," J. Chem. Phys. 152, 184108 (2020).

${ }^{94}$ R. McLaren, S. A. C. Clark, I. Ishii, and A. P. Hitchcock, "Absolute oscillator strengths from K-shell electron-energy-loss spectra of the fluoroethenes and 1,3-perfluorobutadiene," Phys. Rev. A 36, 1683-1701 (1987).

${ }^{95}$ B. T. Pickup and O. Goscinski, "Direct calculation of ionization energies," Mol. Phys. 26, 1013-1035 (1973).

${ }^{96}$ S. Coriani, O. Christiansen, T. Fransson, and P. Norman, "Coupled-cluster response theory for near-edge X-ray-absorption fine structure of atoms and molecules," Phys. Rev. A 85 (2012).

${ }^{97}$ A. Holme, K. J. Børve, L. J. Sæthre, and T. D. Thomas, "Accuracy of calculated chemical shifts in carbon 1s ionization energies from single-reference ab initio methods and density functional theory," J. Chem. Theory Comput. 7, 4104-4114 (2011).

${ }^{98} \mathrm{~T}$. Fransson, $X$-ray spectroscopies through damped linear response theory, $\mathrm{Ph} . D$. thesis, Linköping University (2016). 